\title{
Euh le saviez-vous ? le rôle des (dis)fluences en contexte interactionnel : étude exploratoire et qualitative
}

\author{
Loulou Kosmala \\ Université Sorbonne Nouvelle - Paris III. Paris, France
}

Résumé. Cette étude exploratoire s'inscrit dans un projet d'analyse des (dis)fluences sur un corpus français oral spontané et préparé qui prend en compte les différentes modalités du discours (linguistique, vocal, visuel, gestuel) et qui s'inscrit dans une perspective de linguistique interactionnelle. Les (dis)fluences, caractérisées par une interruption du flux verbal et vocal, ont souvent été strictement analysées du point de vue de la production, et cette étude a pour objectif de dépasser cette approche formelle et de rendre compte de leur ambivalence fonctionnelle et de leur contribution à l'interaction. L'analyse porte sur une paire de locuteurs du corpus, et les résultats préliminaires indiquent un taux de (dis)fluence plus élevé en contexte de discours préparé en classe qu'en conversation semispontanée, ce qui servira de point de départ pour de futures analyses quantitatives plus détaillées sur l'ensemble des données. Cet article repose sur des analyses qualitatives fines d'un extrait de séquence narrative humoristique qui soulignent la dimension interactionnelle des (dis)fluences et la manière dont elles peuvent être employées par les locuteurs à des fins discursives et rhétoriques dans le cadre de tâche narrative.

\begin{abstract}
Uh did you know? The role of (dis)fluencies in interactional contexts: an exploratory and qualitative study. The present study is part of a research project conducted on (dis)fluencies in a French oral corpus of spontaneous and prepared speech which takes into account the different modalities of discourse (linguistic, vocal, visual, and gestural). (Dis)fluencies, which are characterized by an interruption of the vocal and verbal channel, have often been strictly analyzed from a production perspective. Grounded in an interactional approach to language, the aim of the present study is to go beyond this formal approach and to take into account their functional ambivalence and their contribution to the interaction. The analysis is taken from one pair of the corpus, and the preliminary results indicate a higher rate of (dis)fluencies in prepared speech than in conversation. This finding will be used as a starting point for future quantitative analyses on the data. The point of this article is to focus on qualitative analyses on a video extract taken from a humorous sequence in order to stress out their interactional dimension and the way speakers make use of (dis)fluencies for discursive and rhetorical purposes.
\end{abstract}




\section{Ancrage théorique}

\subsection{Présentation des phénomènes de (dis)fluence et leur intérêt en linguistique interactionnelle}

En interaction orale, les locuteurs doivent constamment planifier leurs énoncés en réfléchissant à ce qu'ils vont dire et à la manière de le dire (Clark, 2002) tout en s'ajustant à l'autre. Ceci est constitutif de la parole spontanée : les locuteurs traitent l'information contenue dans l'interaction en la codant et la décodant de manière simultanée. Par conséquent, leurs énoncés sont souvent affectés par des marques d'hésitation comme euh ou eum, des marqueurs de discours (tu vois) mais aussi des répétitions ou autointerruptions, des longues pauses silencieuses, et des auto-corrections immédiates. Cet article porte sur ces phénomènes langagiers dits de (dis)fluence. Le terme (dis)fluence avec des parenthèses sera adopté (suivant la terminologie de Crible et al. 2019 et Götz, 2013) afin de souligner leur polyvalence : les mêmes formes peuvent être à la fois des marques de « fluence » (qui rétablissent la fluence du discours), ou de « disfluence » (qui interrompent et perturbent le flot de la parole) selon de nombreux facteurs contextuels (contexte d'occurrence, durée, place dans l'interaction etc.). Ce terme dépasse donc la dichotomie entre «fluence » et "disfluence » qui est bien trop réductrice puisqu'elle suggère une norme (l'élocution idéale, ideal delivery, Clark 2003 ; la fluence) et une déviation (un écart à la norme, disfluence). Or, l'analyse des corpus oraux et vidéos et la transcription fine des énoncés illustrent bien qu'il est impossible de produire un discours qui ne contient aucune disfluence, et nous verrons par la suite le rôle qu'elles peuvent jouer au sein de l'interaction.

L'étude de la (dis)fluence s'inscrit dans plusieurs domaines. D'abord abordé d'un point de vue pathologique, avec le terme de « disturbances » selon Mahl, (1956), elle souligne un temps d'arrêt, une rupture dans la fluidité de la parole. Chez les psycholinguistes, le terme disfluence (disfluency) rejoint également cette notion d'interruption, mais il souligne aussi l'idée de surcharge cognitive liée aux difficultés des activités langagières (Bortfeld, et al. 2001; Finlayson \& Corley, 2012; Merlo \& Mansur, 2004) Ces phénomènes sont donc plus présents lors de contextes de recherche lexicale (Beattie \& Butterworth, 1979), de planification de longs énoncés (Oviatt, 1995; Shriberg, 1994) et d'incertitude (Bortfeld et al. 2001 ; Schachter et al., 1991). Plusieurs chercheurs se sont donc intéressés à l'étude des (dis)fluences du point de vue de la production, avec l'analyse de la vitesse de la parole et d'élocution, la fréquence, la distribution et durée des pauses (Goldman-Eisler, 1958; Maclay \& Osgood, 1959). Lorsqu'une « erreur » est détectée dans l'énoncé, elle constitue un point d'interruption qui appelle à être réparé, et qui peut s'analyser de manière structurelle avec un Reparandum un Interregnum et Reparans (Clark \& Wasow, 1998; Pallaud et al., 2019). Ces études soulignent l'aspect formel des (dis)fluences, qui a donné lieu à diverses typologies et classifications des marqueurs (voir Lickley, 2015). Ces formes vocales et morpho-syntaxiques peuvent également être détectées dans les corpus oraux de manière automatique dans le cadre de la reconnaissance de la parole, et certaines technologies permettent de les supprimer complètement afin de faciliter la transcription des données (Kaushik et al., 2010). Mais que serait le discours oral sans ces formes, constitutives de la parole spontanée? Certains chercheurs s'efforcent justement de reconstituer des marques de la parole spontanée comme le rire ou les pauses remplies dans les systèmes de dialogue des robots et intelligences artificielles afin de rendre leurs énoncés plus naturels (Kawahara, 2019). Cela souligne l'aspect inextricablement humain et social des (dis)fluences.

Si l'on s'éloigne donc de cette première perspective des (dis)fluencesn strictement formelle et qu'on les considère comme des événements communicatifs, elles prennent une dimension d'autant plus enrichissante et complexe. Comme le souligne Duez (2001, p. 12) : 
« D'une part, elles interrompent le flot de la parole et peuvent rompre l'intégrité de certaines des unités de la parole, d'autre part ce sont des marques de spontanéité qui peuvent apparaître comme telles à l'auditeur »

Les (dis)fluences jouent donc une sorte de double rôle : d'une part, de par leur présence, l'énoncé est temporairement suspendu ou interrompu; d'autre part, elles permettent aux locuteurs de rétablir la fluence communicationnelle des énoncés. Il est donc essentiel de prendre en compte la place des (dis)fluences dans l'interaction afin de mieux comprendre leur emploi. Certaines études s'y sont intéressées, notamment dans le cadre de l'analyse conversationnelle (Schegloff, 2010; Sacks et al. 1974) où les pauses jouent un rôle dans la gestion du dialogue et la prise du tour de parole. Elles peuvent également indiquer un nonalignement, ou une réponse non préférée (Roberts et al. 2006). D'autres (dis)fluences comme les pauses remplies peuvent aussi être employées comme des outils communicatifs qui servent à attirer l'attention sur soi et à établir le contact (Kjellmer, 2003), ou défendre une idée (Tottie, 2014). La richesse des fonctions servies par les (dis)fluences souligne donc leur aspect multidimensionnel et polyvalent. Alors qu'un grand nombre d'études se sont majoritairement concentrées sur l'aspect formel des (dis)fluences et leurs contextes de production (Ferreira \& Bailey, 2004; Levelt, 1983; Shriberg, 1994) sans prendre en compte des situations de communication particulières, cet article se concentre sur leur contribution au sein de séquences interactionnelles, ce qui sera complémentaire des approches plus formelles et quantitatives. Par contexte interactionnel, nous entendons des moments de la situation durant lesquels les co-participants marquent leur intersubjectivité dans le cours des pratiques interactionnelles (Kärkkäinen, 2006) : ajustement et prise en compte de l'autre, expression du point de vue et du positionnement etc. Cette étude des (dis)fluences s'inscrit donc dans une approche interactionnelle et multimodale du langage (Goodwin, 2010; Mondada, 2001) qui prend en compte des caractéristiques relevant de différentes modalités (linguistique, vocal, visuel, gestuel) et qui inclut différents niveaux d'analyses (syntaxe et pragmatique). Selon cette approche, les énoncés font le fruit d'une collaboration interactionnelle entre les interlocuteurs (Goodwin, 1981), et les phénomènes étudiés en discours peuvent servir à réaliser et ou accomplir des « actions » (Mondada, 2008). Nous verrons dans nos analyses la manière dont certaines (dis)fluences en contexte permettent de maintenir la fluence communicationnelle de l'interaction, mais aussi d'ajouter une dimension dramatique au discours. Nous verrons aussi que leur emploi peut également être déterminé par les activités conjointes des locuteurs dans le cadre de co-construction. Cet article vise donc à mieux saisir le rôle des (dis)fluences en interaction spontanée en prenant en compte leurs caractéristiques visuo-gestuelles, en dépassant donc la perspective formelle et verbale adoptée par un grand nombre d'études. Pour ce faire, nous nous intéresserons plus particulièrement aux contextes d'occurrence des (dis)fluences et à leur distribution locale, en favorisant des analyses qualitatives fines d'extraits vidéo, en nous inscrivant donc également dans une perspective d'Analyse Conversationelle (Schegloff et al. 1974). L'analyse portera sur des données enregistrées en contexte naturel (en classe, à l'université) afin de souligner l'importance de l'étude des activités des participants lors de naturally occurring interactions (voir section 4).

\subsection{L'importance de l'apport multimodal des (dis)fluences}

Les phénomènes de (dis)fluence sont un sujet d'étude vaste et complexe, regroupant plusieurs niveaux d'analyse (prosodique, acoustique, syntaxique, phonologique etc.). Nous nous intéresserons ici plus particulièrement à leurs caractéristiques visuo-gestuelles (gestes, expressions faciales et direction du regard). Certaines études se sont intéressées à la question (voir Graziano \& Gullberg, 2013, 2018; Kosmala et al., 2019; Seyfeddinipur, 2006; Seyfeddinipur \& Kita, 2001; Tellier, et al., 2013) et ont notamment montré un lien temporel entre la suspension de la parole et celle des gestes, puisque les locuteurs avaient 
tendance à suspendre ou interrompre leurs gestes lorsqu'ils produisaient des marqueurs de (dis)fluences. La notion de (dis)fluence et d'interruption se traduirait donc sur plusieurs modalités. Pourtant, l'analyse des manifestations gestuelles pendant les (dis)fluences reste peu explorée, et cela peut être expliqué entre autres par le faible pourcentage de gestes qui les accompagnent (Christenfeld et al., 1991; Kosmala et al. 2019). Néanmoins, l'analyse des gestes et du regard lors des (dis)fluences peut permettre de déceler leur dimension pragmatique au sein du discours, et également d'évaluer leurs différents niveaux de DISfluence (Kosmala et al. 2019). Une (dis)fluence qui est accompagnée d'un geste interactionnel (Bavelas et al., 1992) avec le regard tourné vers l'interlocuteur aura un rôle très différent de celle accompagnée d'un geste cyclique (Ladewig, 2014) avec le regard détourné. Dans le premier cas, la (dis)fluence ne traduit pas forcément un problème de production, mais peut au contraire contribuer à la fluence communicationnelle de l'interaction, alors que dans le deuxième cas elle pourrait signaler l'activité langagière de l'interlocuteur (ex. recherche lexicale) afin de faciliter sa production. En analysant la présence des gestes lors des pauses en interactions natif / non-natif, Stam \& Tellier (2017) ont opté pour trois catégories fonctionnelles de gestes : (1) production oriented, facilitant la production des locuteurs, (2) interaction oriented, en lien avec les tours de parole et l'organisation de l'interaction, et (3) comprehension oriented, qui facilitent la compréhension des énoncés pour les interlocuteurs. Elles ont par ailleurs montré que les gestes employés durant les pauses renforçaient la dimension didactique de certaines stratégies de sens dans le cas de gestes comprehension oriented.

L'intégration de l'analyse des gestes en interaction est également essentielle puisqu'ils peuvent jouer un rôle dans la séquentialité de l'interaction avec les tours de parole (Mondada, 2007; Streeck, 2008), et indiquer les aspects d'une force illocutoire, ou un positionnement (Kendon, 1995, 2004). La prise en compte de toutes ces ressources sémiotiques est donc indispensable afin de mieux comprendre le rôle des (dis)fluences en interaction, permettant ainsi de révéler des mécanismes non observables autrement.

\section{Corpus et méthodes}

\subsection{Présentation du corpus DisReg}

Les données utilisées dans le cadre de cette étude proviennent d'un corpus vidéo collecté en automne 2018 à l'université de Paris III - Sorbonne Nouvelle. Il contient des enregistrements de douze étudiants francophones de $3^{\text {ème }}$ et $2^{\text {ème }}$ année de licence de littérature française et comparée qui ont donné leur accord pour être d'abord filmés en classe dans le cadre d'une évaluation orale, et ensuite par paires dans une salle de classe lors d'une discussion semi guidée. La première partie du corpus contient donc des enregistrements faits en classe (Figure 1). L'investigatrice tenait la caméra en face de l'étudiant (ou des étudiants s'ils faisaient la présentation par paire) durant leurs présentations orales qui durent de 15 à 50 minutes. Ils avaient plusieurs supports à leur disposition (notes papier, tableau, ordinateur, parfois projection powerpoint). Les mêmes étudiants étaient ensuite filmés à nouveau par paire dans une salle de classe (Fig. 2) lors d'une conversation semi-guidée. Les deux étudiants se connaissaient bien, ils partageaient des cours ensemble, et étaient parfois amis en dehors de l'université. Lors du deuxième enregistrement, ils disposaient d'un morceau de papier où étaient écrits quelques sujets de conversation (dernier film vu au cinéma, anecdote drôle à l'université, utilisation des réseaux sociaux etc.) mais ils étaient également libres de parler de ce qui leur plaisait. La durée des échanges varie entre 15 et 25 minutes.

Le but de cette étude est de comparer l'emploi des (dis)fluences dans des contextes de communication et registres très différents, certes tous deux dans le même cadre 
institutionnel (l'université) mais dans des situations qui s'opposent. D'une part un cadre formel dans une situation de discours préparé (présentation orale préparée à la maison), et d'autre part un cadre plus informel lors d'interactions semi spontanées. De plus, ces enregistrements se rapprochent plutôt bien des pratiques de la vie de tous les jours puisque les étudiants ont souvent des exposés oraux à faire dans le cadre de la licence ; et même si les interactions sont semi-guidées, elles ont lieu à l'université, un lieu de socialisation. Cependant, la place de la caméra a également une incidence sur le comportement des participants, puisque ça les pousse parfois à se mettre en scène, ou au contraire ça peut les rendre mal à l'aise. C'est un aspect que nous prendrons en compte dans l'analyse qualitative (section 4).

Ces données ont été récoltées dans le cadre d'une étude quantitative plus large qui est actuellement en cours de traitement. Dans cet article, nous présenterons des analyses tirées d'un extrait d'une paire du corpus en nous focalisant plus particulièrement sur la partie conversationnelle afin de rendre compte de l'emploi des (dis)fluences en contexte. La paire choisie est composée de deux locuteurs français, camarades de classe et amis, que nous nommerons Paul et Paula ${ }^{i}$ dans le cadre de cette étude.
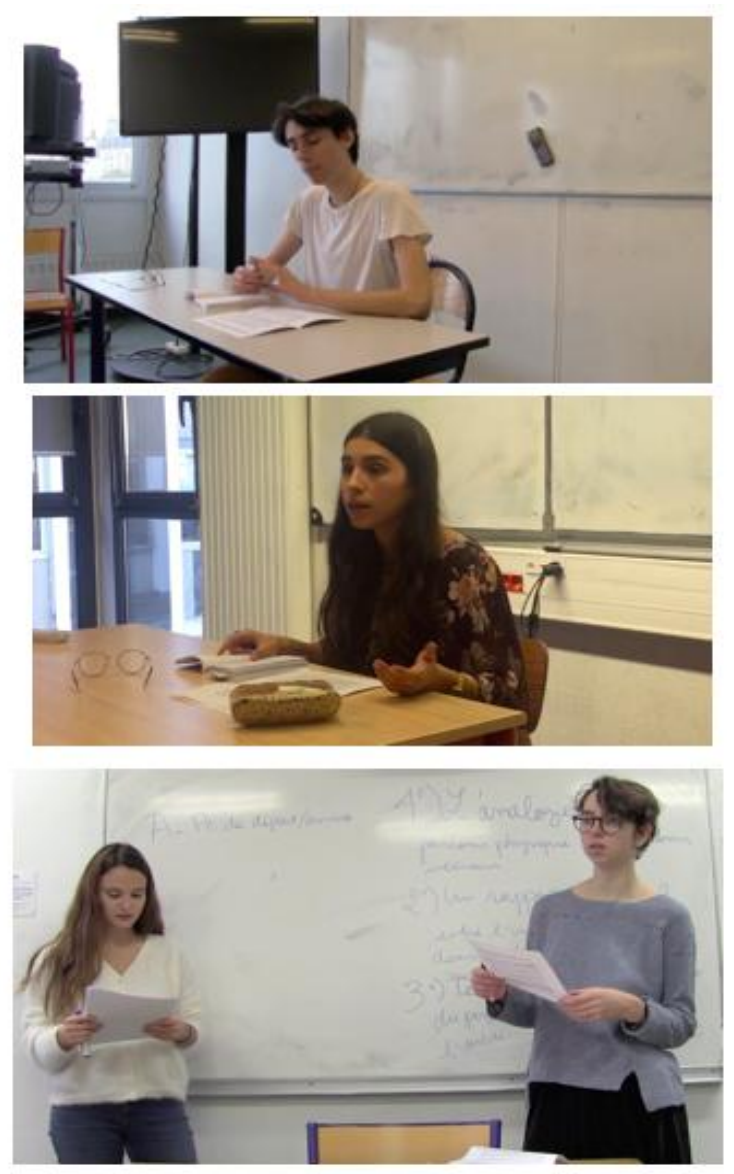

Fig. 1. Enregistrement des étudiants en classe 

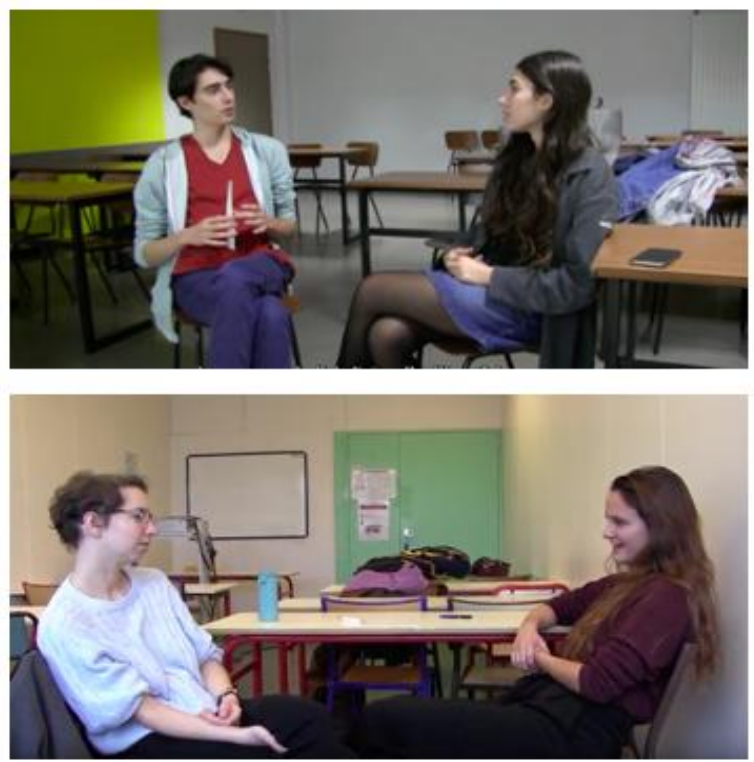

Fig. 2. Interactions semi-spontanées

\section{Méthode d'analyse des (dis)fluences}

Les méthodes utilisées pour cette étude sont tirées de plusieurs travaux portant sur les phénomènes de (dis)fluence en anglais et en français dans le cadre d'interactions tandems (Kosmala \& Morgenstern, 2017; Kosmala et al. 2019 ; Kosmala 2019). L'objectif est de reproduire cette méthode de codage sur de nouvelles données, afin d'explorer l'ambivalence fonctionnelle de ces phénomènes à travers les langues et les contextes de communication. L'analyse consiste d'abord à relever tous les marqueurs de (dis)fluence répertoriés dans la littérature (voir Shriberg, 1994 et Lickley 2015), soit les pauses silencieuses, les pauses remplies (euh et eum), les auto-corrections, les répétitions nonlexicales, les allongements de syllabe, les mots tronqués, les termes explicites d'édition (voir (Crible, 2017) et les sons non-lexicaux (nasalisations, les clics etc.). Nous partons du principe que ces formes, qui constituent la «disfluence », certes de manière strictement formelle a priori, peuvent également être analysées au sein de séquences particulières (comme Heritage, (2002) qui a travaillé sur oh par exemple). C'est justement en étudiant ces formes en contexte que l'on peut déceler leur rôle en interaction. L'analyse visuogestuelle des (dis)fluences est donc également essentielle dans le cadre de cette analyse. Cela inclut la prise en compte de la direction du regard (vers l'interlocuteur ou détourné) les expressions faciales (sourire, froncement de sourcils etc.) et les gestes manuels (voir Kosmala 2019 ; Kosmala et al. 2019 pour une description plus détaillée). La classification utilisée pour l'analyse des gestes qui accompagnent les (dis)fluences est tirée de plusieurs typologies fonctionnelles existantes (Kendon, 2004 ; Muller, 1998, cité par Cienki, 2005; Gullberg, 2011) soit les gestes représentationnels, les gestes deictiques-anaphoriques, et les gestes pragmatiques (regroupant les gestes discursifs et les gestes interactifs). L'ensemble des productions a été transcrit et annoté à l'aide du logiciel ELAN (Sloetjes \& Wittenburg, 2008). 


\section{Analyses}

\subsection{Distribution des (dis)fluences sur une partie du corpus}

Sur 10 minutes tirées du corpus environ, 325 occurrences de (dis)fluences ont été relevées, avec 236 occurrences pour la partie préparée en classe et 89 pour la partie spontanée. En moyenne, Paul et Paula ont produit 7,5 (dis)fluences tous les 100 mots en classe et 4,6 tous les 100 mots en conversation spontanée, avec quelques différences individuelles pour la partie préparée : sur quelques minutes, Paul en a produit 142 lors de son exposé oral, alors que Paula en a produit 94. Il semblerait que les locuteurs ont eu davantage tendance à produire des (dis)fluences en classe qu'en conversation spontanée. Cela peut s'expliquer par le fait que les enregistrements en classe peuvent présenter des situations de stress et d'anxiété, ce qui peut avoir une influence sur la production des (dis)fluences (Mahl, 1956). Ces résultats préliminaires servent de point de départ pour de prochaines analyses quantitatives sur une plus grosse portion du corpus. Néanmoins, nous préférons ici nous concentrer sur une étude de cas qui permet de montrer l'emploi stratégique des (dis)fluences dans le cadre d'une séquence humoristique, ce qui a plus d'intérêt dans notre étude d'analyse du discours.

\subsection{Analyses qualitatives: le rôle des (dis)fluences lors de séquences humoristiques}

L'objectif principal de cet article est de rendre compte de la contribution des (dis)fluences en contexte interactionnel en analysant quelques occurrences dans une séquence précise. Nous nous inscrivons donc dans une perspective d'analyse conversationnelle qui se concentre sur des analyses multimodales qualitatives sur une petite portion du corpus. L'analyse qualitative permet de rendre compte de l'usage d'un phénomène dans un contexte de socialisation particulier, donc nous avons choisi ici d'étudier une séquence tirée de la partie conversation du corpus entre Paul et Paula. Dans cette séquence, Paul et Paula essaient de trouver " une anecdote marrante à raconter en lien avec l'université » (sujet écrit sur le bout de papier) et c'est Paul qui commence sa narration. Cette séquence a été choisie car elle présente un certain intérêt : Paul ne fait pas que raconter une anecdote, il se met également en scène en jouant le rôle d'un narrateur humoristique. Cette «tâche narrative » présente des enjeux discursifs et rhétoriques pour les locuteurs, puisqu'ils ont comme « mission» de raconter une histoire qui fera rire l'autre. On observe d'ailleurs à la fin de l'extrait comme Paula le remarque (ligne 39, ça c'était une anecdote marrante) que la tâche a été achevée avec succès par Paul. Alors que les (dis)fluences ont souvent été proscrites par les rhétoriciens car elles reflètent un parler jeune ou une mauvaise maîtrise de la langue (Jean \& Fox Tree 2007) cette analyse montrera qu'elles peuvent contribuer à la rhétorique du discours. L'enjeu ici est donc de montrer que les (dis)fluences ne traduisent pas seulement une interruption du flux vocal ou verbal en lien avec des difficultés de production. Nous verrons la manière dont Paul mobilise également plusieurs ressources sémiotiques afin de raconter son histoire et la rendre comique.

Transcription verbatim de l'extrait ${ }^{\mathrm{ii}}$

7 *PAULA: \&euh une anecdote t'en as pas une à dire avant que je t'en trouve une ?

8 *PAUL: \&eu:m $(0.400)<\mathrm{clic}>\& \mathrm{~mm}$.

9 *PAULA: sur l'université.

10 *PAUL: \&eum \&ah si j'en ai une elle est très très précise.

11 *PAULA: ok. 
12 *PAUL: \&eum le [//] la [/] la personne avec qui j'ai dû \&eum \&euh faire des bras de fer pour être admis à paris+3 en 11 .

13 *PAUL: \&euh c'était un secrétaire alors c'était l'année où \&euh $+/ /$.

14 *PAUL: le saviez-vous ?

$15 *$ PAUL: l'année dernière ils ont viré $<$ tous les $>$ [=! rit] [/] tous les secrétaires.

16 *PAUL: nous a dit \&u [//] une prof de linguistique \&euh au [/] au premier semestre.

17 *PAUL: elle a dit +* \&ah j'adore le fonctionnement de XX vraiment c'est génial+*.

18 *PAUL: on a [//] on s'est plaint en fin d'année dernière donc en fin de ma L1 parce-que l'administration était beaucoup trop \&euh lente et que les gens étaient pas réactifs etc machin.

19 *PAUL: et du coup la présidence a dit *+ niquel on a trouvé une solution on va virer tout le monde $+*[=$ ! rit $]$ et ça va aller beaucoup mieux.

20 *PAULA: $+<$ ah bah génial.

21 *PAUL: et du coup ils ont viré tout le monde et réembauché des gens [=! rit] voilà.

22 *PAUL: et \&euh [/] et < guess what> [\%en] ça (n')a pas changé grand-chose.

23 *PAUL: mais \&euh [/] mais [/] mais surtout qu'en plus c'est complètement con parcequ'il faut un temps de formation des gens après bon bref.

\section{4 *PAUL: donc du coup ce mec qui malheureusement a été viré après parce-qu'il était génial qui s'appelait $\mathrm{XX}$.}

25 *PAUL: <clic $>(0.400)$ salut.

26 *PAUL: \&euh il \&euh [/] il se baladait avec des \&eum [/] des \&euh je sais pas si tu vois les [/] les [/] le:es [//] des gants de pieds.

27 *PAULA: $+<\&$ ah oui je vois très bien c'est celles avec les orteils.

28 *PAUL: c'est des chaussures avec le:es \&euh voilà.

29 *PAUL: avec les orteils.

30 *PAUL: et il avait des [//] ces chaussures-là.

31 *PAUL: alors \&j (0.430) au début je l'avais vu que à son bureau donc il était assis à son bureau etc. donc je voyais son [/] son buste comme ça et on parlait et tout.

32 *PAUL: et un jour il est [//] il m'a dit +* \&oh ben accompagnez moi dans le couloir \&euh machin je vais faire un truc et tout on va parler*+.

33 *PAUL: et il s'est levé il a fait le tour de son bureau et j'ai vu qu'il avait de:es [//] les [/] les ga:ants de pieds là un espèce de plastique xx.

34 *PAUL: [= ! rit $]$ genre \&ah [= ! rit $]$ gardez ma dignité surtout.

35 *PAUL: [= ! rit] et du coup j'ai continué à parler \&ah oui bien sur \&mm oui très bien etc $\& \mathrm{~mm}$ t'as des gants de pieds c'est trop bizarre.

36 *PAULA: c'est tellement drôle.

37 *PAUL: voilà.

38 *PAULA: d'accord.

39 *PAULA: ça c'était une anecdote marrante. 
En vue du grand nombre de (dis)fluences présentes dans cet extrait $(\mathrm{N}=48)$, nous avons sélectionné trois moments dans l'interaction (en gras) qui ont suscité notre intérêt dans le cadre de cet article.

\subsubsection{Emploi de l'auto-interruption à des fins humoristiques: stratégie interactionnelle et reprise d'un genre}

Dans la séquence sélectionnée, Paul et Paula réfléchissent à une anecdote drôle à raconter, afin de se conformer à ce qui leur a été proposé sur un des sujets écrits sur le bout de papier. Cette séquence commence au bout de quatre minutes d'enregistrement. Après un moment de réflexion, (1. 7-9), Paul finit par en trouver une (1. 10) et se lance donc dans une « tâche narrative » qui contient un événement problématique (Ochs \& Capps, 2001) qui le mènera au point culminant de son récit (« complication » Labov 1997) (voir la section 4.1.3). Le premier moment qui nous intéresse apparaît à la ligne 13. Paul débute son histoire en introduisant le «protagoniste » de son histoire, un personnel administratif, qu'il décrit tout d'abord comme étant « la personne avec qui j'ai dû \&eum \&euh faire des bras de fer pour être admis à Paris 3 [l'université] ». Mais avant de parler plus longuement de cette personne, il décide de faire une digression et de parler du fonctionnement de l'université (lignes 13-25). On observe donc une rupture au sein de son discours (1.13-14), à la fois d'un point de vue narratif, puisqu'il change brusquement le cours de son histoire, mais aussi d'un point de vue syntaxique, puisqu'il interrompt sa phrase (1.13, marqué par la pause remplie et l'auto-interruption à la fin de l'énoncé) et en commence une autre le saviez-vous, sous la forme d'une insertion parenthétique. Les auto-interruptions sont généralement les marques de (dis)fluence les plus «sévères» puisqu'elles n'établissent pas de lien sémantique ni syntaxique avec ce qui précède, à la différence des auto-corrections immédiates (Crible, 2017). D'un point de vue strictement formel, nous avons donc une rupture de la fluidité de l'énoncé. Seulement, d'un point de vue discursif et interactionnel, la (dis)fluence joue un tout autre rôle. L'insertion parenthétique qui suit l'auto-interruption joue un rôle "modélisateur" (Forget, 2000) puisqu'elle lui permet de revenir sur ce qu'il vient de dire pour préciser le fonctionnement de l'université. Mais elle permet surtout de contribuer à l'aspect comique de son discours en reprenant la fameuse phrase " Le saviezvous? ». Cette expression figée, que l'on retrouve souvent dans des chroniques, jingles, ou plateaux télévisés, vise en général à traiter une information factuelle de manière vulgarisée. En produisant cette phrase, l'énonciateur adopte un autre genre où il semble jouer le rôle d'un présentateur de télévision qui présente un fait amusant, et qui sort des "punchlines ", comme "guess what» (1.22). Il s'adresse alors à Paula comme si elle faisait partie d'un public imaginaire sur un plateau télévisé. Cette stratégie interactionnelle se traduit également sur le plan non-verbal, puisqu'il se tourne vers son interlocutrice et produit un geste de pointage interactif lorsqu'il produit l'insertion parenthétique (Figure 3). Cette autointerruption suivie de l'insertion parenthétique, ne constitue donc en aucun cas une marque de DISfluence dans le sens strict du terme, puisqu'elle permet de maintenir la fluence communicationnelle du discours, et d'y ajouter une dimension comique. C'est justement par le biais de l'auto-interruption que Paul permet de mieux consolider son histoire, en racontant le fonctionnement de l'université qui a mené au licenciement de son « protagoniste ». 


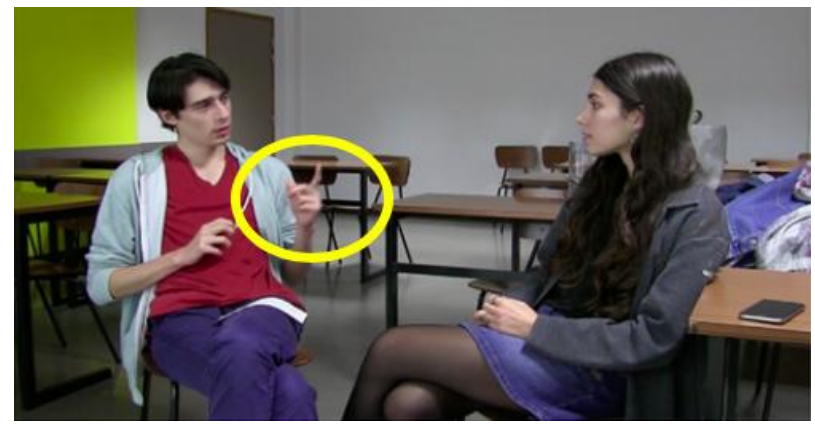

Fig. 3. Geste interactif lors d'une insertion parenthétique

\subsubsection{Emploi pragmatique de la pause : réintroduction du protagoniste}

Après avoir raconté à Paula l'incident lié à l'université, Paul réintroduit le protagoniste de son histoire en soulignant qu'il a été licencié, et en prononçant son nom (anonymisé, ligne 24), ce qui fait de lui une figure presque héroïque. Cet aspect-là est accentué à la ligne 25 quand Paul décide de le saluer pour lui rendre un hommage, comme s'il était présent dans la pièce. A nouveau, Paul ne fait pas que raconter une anecdote, il la met en scène, ce qui accentue d'autant plus la dimension comique et dramatique de son récit. Il produit par ailleurs à nouveau un geste interactif mais cette fois-ci avec l'index levé et les yeux baissés, en adoptant une posture presque solennelle (Fig 4.) Ce geste semble être employé afin d'attirer l'attention sur son protagoniste, pour lui accorder de l'importance et le réintroduire au sein du récit. Ce ton solennel est également marqué par l'emploi de la pause silencieuse (de 400 millisecondes) et du clic (voir Wright, 2007) avant de dire salut. Ici la pause silencieuse et le clic ne marquent pas seulement une suspension du flux vocal, ils permettent également à Paul de mettre l'emphase sur ce qui suit, ce qui rend son «hommage» au protagoniste d'autant plus cérémonieux. A nouveau, Paul mobilise plusieurs ressources sémiotiques (geste et regard) qui accompagnent les (dis)fluences afin de dramatiser son récit.

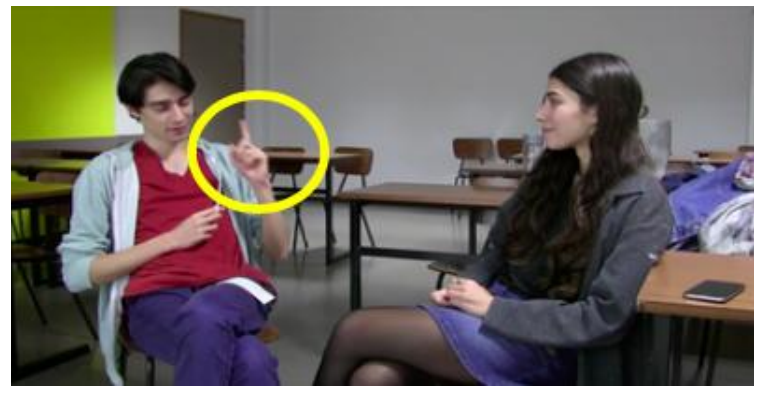

Fig. 4. Posture quasi solennelle du locuteur lors de la pause silencieuse

La dimension polyvalente des (dis)fluences est également mise en exergue ici, car la pause et le clic apparaissent dans un contexte en lien avec la stratégie interactionnelle du locuteur. Elles contribuent parfaitement à l'aspect humoristique de son récit, qui ici repose sur des éléments de dramatisation. De plus, la pause silence marque également un changement de co-énonciateur puisqu'il ne s'adresse plus à Paula à cet instant-là mais à ce protagoniste qu'il salue. C'est d'ailleurs par le biais de la pause qu'il parvient à ajuster son point de vue. La notion de (dis)fluence dépasse donc la perspective strictement structurelle des énoncés mais prend également en compte la dimension rhétorique et communicationnelle du discours. 


\subsubsection{Emploi multimodal des (dis)fluences dans un contexte de co-construction du sens : point culminant du récit}

Nous nous tournons à présent vers la fin de l'extrait (1.26-29) où Paul fait à nouveau recours à des (dis)fluences, mais cette fois ci dans un contexte de co-construction. Paul semble avoir quitté son rôle de présentateur humoristique, et il se tourne à nouveau vers Paula afin de lui décrire les chaussures plutôt insolites du protagoniste. Ces chaussures, ces «gants de pied » représentent le point culminant de son récit car c'est sur l'aspect incongru de ces chaussures que repose son anecdote humoristique. À la ligne 26, il produit une série de (dis)fluences (deux pauses remplies, une répétition, un allongement syllabique etc.) et il produit également en même temps un geste iconique représentationnel en tendant son bras et sa main devant lui, positionnée de sorte à ce qu'elle soit visible par son interlocutrice. Paula a d'ailleurs les yeux tournés vers son geste, et sourit légèrement (Figure 5).

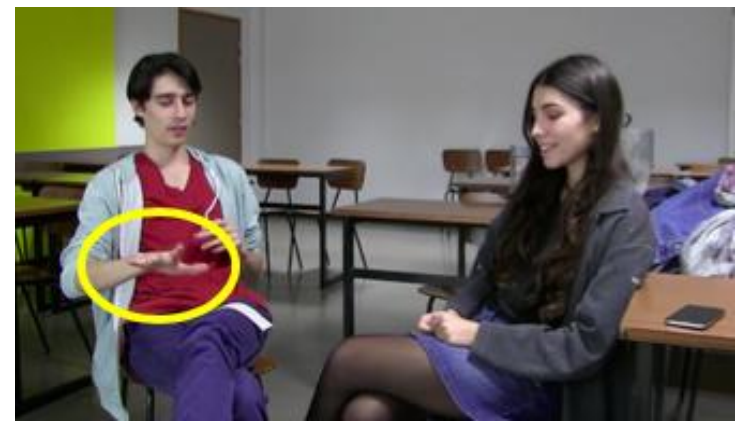

Fig. 5. Geste représentationnel pour captiver l'attention de l'interlocutrice

Ici Paul a recours à des (dis)fluences et un geste représentationnel qui lui permettent à la fois de captiver l'attention de Paula sur ce point du récit, et aussi de rendre visible l'aspect iconique et humoristique de ces chaussures atypiques. De plus, ce geste repose sur la notion de common ground (Clark, 1996) puisque les deux participants de la conversation construisent ensemble le sens du terme en se fondant sur leur savoir partagé. Ces chaussures excentriques, outre leur originalité, ne sont pas très communes, donc Paul veut également s'assurer que sa partenaire comprenne de quoi il s'agit par le biais d'une expression intersubjective je sais pas si tu vois (l.26). On observe également dans cet extrait un cas de résonnance dialogique qui indique un alignement syntaxique (Du Bois, 2007) car Paula complète l'énoncé de Paul en précisant que ce sont des chaussures où l'on voit les orteils (1. 29). C'est par ailleurs peut-être cet aspect saillant que Paul a voulu représenter à travers son geste en faisant apparaitre distinctement ses doigts (Figure 6). Ce geste est d'ailleurs repris un peu plus tard dans l'interaction quand Paul raconte plus en détail la manière dont il s'est retrouvé dans le bureau de son protagoniste et qu'il a aperçu ses chaussures (1.33). Cette représentation gestuelle fait donc également l'objet d'une construction de chaîne de référence multimodale, où le référent n'est pas seulement repris oralement (de:es [//] les [/] les ga:ants de pieds là, 1. 33) mais également par le biais d'une reprise gestuelle, ce qui pourrait indiquer un statut d'information connue (Debras \& Beaupoil-Hourdel, 2019).

Cet exemple a montré que l'emploi des (dis)fluences en synchronie avec des manifestations gestuelles dans certains contextes interactionnels peut également permettre aux locuteurs de gérer leurs relations interpersonnelles. Alors que dans les deux premiers exemples Paul jouait le rôle quasi désincarné d'un narrateur humoristique, il redevient participant de l'interaction en sollicitant l'attention de sa partenaire qui participe à la coconstruction de sa narration. À la différence des deux exemples précédents, où Paul employait des gestes interactifs à des fins humoristiques et dramatiques, ici il déploie 
plusieurs gestes référentiels pour exprimer visuellement l'aspect incongru des gants de pieds, et pour co-construire le sens avec son interlocutrice. A nouveau, les (dis)fluences que l'on observe ici ne traduisent en aucun cas des difficultés de production et une rupture au sein de son discours, elles permettent au contraire de maintenir la fluence communicationnelle de l'interaction.

\section{Synthèse}

Cette étude qualitative et exploratoire avait pour objectif de présenter une méthode d'analyse des phénomènes de (dis)fluence qui prenne en compte leurs caractéristiques formelles multimodales (forme du marqueur et caractéristiques visuogestuelles) et leur dimension interactionnelle au sein de pratiques langagières contextualisées (présentation individuelle en classe et échanges portant sur l'université). Cette étude multimodale a permis de mettre en lumière la dimension interactionnelle des (dis)fluences en rendant compte de leurs différents usages lors d'une séquence tirée du corpus. Le locuteur, par le biais de (dis)fluences et autres ressources visuogestuelles, a opté pour plusieurs stratégies interactionnelles afin de présenter à son interlocutrice un récit humoristique. Les trois analyses ont montré la richesse d'emploi des (dis)fluences qui peuvent être utilisées à des fins humoristiques (4.2.1) et rhétoriques (4.2.2.), ou pour captiver l'attention de l'interlocutrice (4.2.3) dans un contexte de co-construction. Plusieurs manifestations gestuelles ont également été déployées lors des (dis)fluences, notamment des gestes interactifs (4.2.1 et 4.2.2) et référentiels (4.2.3), ce qui souligne l'aspect multimodal de ces phénomènes. La prise en compte des multiples ressources sémiotiques permet donc de déceler le rôle pragmatique des (dis)fluences en discours. Les phénomènes de (dis)fluence ne devraient donc pas être uniquement considérées du point de vue de la production vocale et verbale, mais également du point de vue de l'interaction afin d'en tirer des résultats complémentaires et observables dans les analyses qualitatives multimodales.

Les résultats préliminaires tirés d'un petit bout du corpus ont montré que les (dis)fluences sont fréquentes mais également très variées, car elles dépendent fortement du contexte communicationnel (plus fréquentes en classe qu'en conversation). Ces résultats servent de point de départ pour notre future étude quantitative sur une plus grosse partie du corpus. Néanmoins les analyses qualitatives ont permis d'illustrer la richesse de leur emploi en contexte, ce qui souligne le besoin d'articuler les approches quantitatives et qualitatives.

Je tiens à remercier Aliyah Morgenstern et Maria Candea pour la relecture de cet article, ainsi que Pauline Beaupoil-Hourdel, Camille Debras, Elinor Ochs, Sandro Duranti, et les autres membres du QualiLab pour leurs retours sur mes analyses qualitatives.

\section{Conventions CHAT pour la transcription des (dis)fluences}

Tirés du manuel CLAN, disponible sur http://childes.psy.cmu.edu

$+/ /$ : auto-interruption par le locuteur

$+/$ : interruption par quelqu'un d'autre

+ " : discours direct, citation

[/] répétition sans reprise

$[/ /]$ reprise avec correction

$[/ / /]$ reprise avec reformulation

$[=!$ rit $]$ événements paralinguistiques

(0.400) : pause avec indication de durée ( 0.4 secondes)

$+<$ chevauchements

$\&$ : symbole devant les pauses remplies et les interjections 


\section{Références bibliographiques}

Bavelas, J. B., Chovil, N., Lawrie, D. A., \& Wade, A. (1992). Interactive gestures. Discourse Processes, 15(4), 469-489.

Beattie, G. W., \& Butterworth, B. L. (1979). Contextual probability and word frequency as determinants of pauses and errors in spontaneous speech. Language and Speech, 22(3), 201-211.

Betz, S., \& Gambino, S. L. (2016). Are we all disfluent in our own special way and should dialogue systems also be? Elektronische Sprachsignalverarbeitung (ESSV) 2016, 81.

Bortfeld, H., Leon, S. D., Bloom, J. E., Schober, M. F., \& Brennan, S. E. (2001). Disfluency Rates in Conversation: Effects of Age, Relationship, Topic, Role, and Gender. Language and Speech, 44(2), 123-147.

Christenfeld, N., Schachter, S., \& Bilous, F. (1991). Filled pauses and gestures: It's not coincidence. Journal of Psycholinguistic Research, 20(1), 1-10.

Cienki, A. (2005). Image schemas and gesture. From Perception to Meaning: Image Schemas in Cognitive Linguistics, 29, 421-442.

Clark, H. H. (1996). Using language. Cambridge: Cambridge University Press.

Clark, H. H. (2002). Speaking in time. Speech Communication, 36(1), 5-13.

Clark, H. H., \& Wasow, T. (1998). Repeating words in spontaneous speech. Cognitive Psychology, 37(3), 201-242.

Corley, M., \& Stewart, O. W. (2008). Hesitation disfluencies in spontaneous speech: The meaning of um. Language and Linguistics Compass, 2(4), 589-602.

Crible, L. (2017). Discourse markers and (dis)fluency across registers: A contrastive usage-based study in English and French (UCL - Université Catholique de Louvain).

Debras, C., \& Beaupoil-Hourdel, P. (2019). Gestualité et construction des chaînes de référence dans un corpus d'interactions tandem. Cahiers de Praxématique, (72).

Du Bois, J. W. (2007). The stance triangle. Stancetaking in Discourse: Subjectivity, Evaluation, Interaction, 164(3), 139-182.

Duez, D. (1991). La pause dans la parole de l'homme politique. Editions du Centre national de la recherche scientifique.

Duez, D. (2001). Signification des hésitations dans la parole spontanée. Revue Parole, 17-18.

Ferré, G. (2008). Récits de femmes Analyse multimodale du récit conversationnel en français: une étude de cas. In Congrès Mondial de Linguistique Française (p. 081). EDP Sciences.

Ferreira, F., \& Bailey, K. G. D. (2004). Disfluencies and human language comprehension. Trends in Cognitive Sciences, 8(5), 231-237.

Finlayson, I. R., \& Corley, M. (2012). Disfluency in dialogue: An intentional signal from the speaker? | SpringerLink. Psychonomic Bulletin \& Review, 19(5), 921-928.

Fox Tree, J. E. F. (2007). Folk notions of um and uh, you know, and like. Text \& Talk-An Interdisciplinary Journal of Language, Discourse Communication Studies, 27(3), 297-314.

Forget, D. (2000). Les insertions parenthétiques. Revue Québécoise de Linguistique, 28(2), 15-28.

Goldman-Eisler, F. (1958). The predictability of words in context and the length of pauses in speech. Language and Speech, 1(3), 226-231.

Goodwin, C. (1981). Conversational Organization: Interaction Between Speakers and Hearers. Academic Press.

Goodwin, C. (2010). Multimodality in human interaction. Calidoscopio, 8(2).

Graziano, M., \& Gullberg, M. (2013). Gesture production and speech fluency in competent speakers and language learners. Presentado En TIGER, Tilburg University, Holanda.

Graziano, M., \& Gullberg, M. (2018). When speech stops, gesture stops: Evidence from developmental and crosslinguistic comparisons. Frontiers in Psychology, 9, 879.

Gullberg, M. (2011). Multilingual multimodality: Communicative difficulties and their solutions in second-language use. Embodied Interaction: Language and Body in the Material World, $137-151$.

Heritage, J. (2002). "Oh"-prefaced responses to assessments: A method of modifying agreement/disagreement. In C. Ford, B. Fox, \& S. Thompson (Eds.), The Language of Turn and Sequence. New York: Oxford University Press. 
Holmes, V. M. (1988). Hesitations and sentence planning. Language and Cognitive Processes, 3(4), $323-361$.

Kärkkäinen, E. (2006). Stance taking in conversation: From subjectivity to intersubjectivity. Text \& Talk-An Interdisciplinary Journal of Language, Discourse Communication Studies, 26(6), 699-731.

Kaushik, M., Trinkle, M., \& Hashemi-Sakhtsari, A. (2010). Automatic detection and removal of disfluencies from spontaneous speech. Proceedings of the Australasian International Conference on Speech Science and Technology (SST), 70.

Kendon, A. (1995). Gestures as illocutionary and discourse structure markers in Southern Italian conversation. Journal of Pragmatics, 23(3), 247-279.

Kendon, A. (2004). Gesture: Visible action as utterance.

Kjellmer, G. (2003). Hesitation. In defence of er and erm. English Studies, 84(2), 170-198.

Kawahara, T. (2019). Spoken dialogue system for a human-like conversational robot ERICA. In 9th International Workshop on Spoken Dialogue System Technology (pp. 65-75). Springer, Singapore.

Kosmala, L. (2019) On the Multifunctionality and Multimodality of Silent Pauses in Native and Nonnative Interactions. Proceedings of the $1^{\text {st }}$ International Seminar on the Foundations of Speech-Pausing Breathing and Voice. University of Southern Denmark.

Kosmala, L., Candea, M., \& Morgenstern, A. (2019). Synchronization of (Dis)fluent Speech and Gesture: A Multimodal Approach to (Dis)fluency. Gesture and Speech in Interaction. Paderborn University.

Kosmala, L., \& Morgenstern, A. (2017). A preliminary study of hesitation phenomena in L1 and L2 productions: A multimodal approach. TMH-QPSR, 37.

Labov, W. (1997). Some Further Steps in Narrative Analysis. Journal of Narrative and Life History 7,: 395-415.

Ladewig, S. H. (2014). Recurrent gestures. In C. Müller, A. Cienki, S. H. Ladewig, D. McNeill, \& S. Tessendorf (Eds.), Body- Language- Communication: An International Handbook on Multimodsality in Human Interaction. Mouton de Gruyter.

Levelt, W. J. (1983). Monitoring and self-repair in speech. In Cognition (Vol. 14, pp. 41-104).

Lickley, R. J. (2015). Fluency and Disfluency. In M. A. Redford (Ed.), The Handbook of Speech Production (p. 445).

Maclay, H., \& Osgood, C. E. (1959). Hesitation phenomena in spontaneous English speech. Word, $15(1), 19-44$.

Mahl, G. F. (1956). Disturbances and silences in the patient's speech in psychotherapy. The Journal of Abnormal and Social Psychology, 53(1), 1.

Merlo, S., \& Mansur, L. L. (2004). Descriptive discourse: Topic familiarity and disfluencies. Journal of Communication Disorders, 37(6), 489-503.

Mondada, L. (2001). Pour une linguistique interactionnelle. Marges Linguistiques, 1, 142-162.

Mondada, L. (2007). Multimodal resources for turn-taking: Pointing and the emergence of possible next speakers. Discourse Studies, 9(2), 194-225.

Mondada, L. (2008). Contributions de la linguistique interactionnelle. Congrès Mondial de Linguistique Française, 073. EDP Sciences.

Ochs, E., \& Capps, L. (2001). Living narrative: Creating lives in everyday storytelling. Harvard University Press.

Oviatt, S. (1995). Predicting spoken disfluencies during human-computer interaction. Computer Speech and Language, 9(1), 19-36.

Pallaud, B., Bertrand, R., Prevot, L., Blache, P., \& Rauzy, S. (2019). Suspensive and Disfluent Self Interruptions in French Language Interactions. Fluency and Disfluency across Languages and Language Varieties, Corpora and Language in Use. Presses Universitaires de Louvain

Roberts, F., Francis, A. L., \& Morgan, M. (2006). The interaction of inter-turn silence with prosodic cues in listener perceptions of "trouble" in conversation. Speech Communication, 48(9), 1079-1093.

Schachter, S., Christenfeld, N., \& Bilous, F. (1991). Speech Disfluency and the Structure of Knowledge. Journal of Personality and Social Psychology, 60(3), 362-367.

Schegloff, E. A. (2010). Some other "uh (m)" s. Discourse Processes, 47(2), 130-174.

Sacks, H., Schegloff, \& E., Jefferson. (1974). A simplest systematics for the organization of turntaking for conversation. Language, 50(4), 696-735.

Seyfeddinipur, M. (Ed.). (2006). Disfluency: Interrupting speech and gesture. 
Seyfeddinipur, M., \& Kita, S. (2001). Gesture as an indicator of early error detection in selfmonitoring of speech. ISCA Tutorial and Research Workshop (ITRW) on Disfluency in Spontaneous Speech.

Shriberg, E. E. (1994). Preliminaries to a Theory of Speech Disfluencies. Thèse de doctorat.

Sloetjes, H., \& Wittenburg, P. (2008). Annotation by category-ELAN and ISO DCR. 6th International Conference on Language Resources and Evaluation (LREC 2008).

Stam, G., \& Tellier, M. (2017). The sound of silence. Why Gesture?: How the Hands Function in Speaking, Thinking and Communicating, 7, 353.

Streeck, J. (2008). Depicting by gesture. Gesture, 8(3), 285-301.

Tellier, M., Stam, G., \& Bigi, B. (2013). Gesturing while pausing in conversation: Selforiented or partner-oriented?". The Combined Meeting of the 10th International Gesture Workshop and the 3rd Gesture and Speech in Interaction Conference, Tillburg (The Netherlands).

Tottie, G. (2011). Uh and Um as sociolinguistic markers in British English. International Journal of Corpus Linguistics, 16(2), 173-197. h

Tottie, G. (2014). On the use of uh and um in American English. Functions of Language, 21(1), 6-29.

Witton-Davies, G. (2014). The study of fluency and its development in monologue and dialogue. Lancaster University, Lancaster. Thèse de doctorat non publiée.

Wright, M. (2007). Clicks as markers of new sequences in English conversation. Proceedings of the 16th International Congress of Phonetic Sciences, 1069-1072. by Jürgen Trouvain and William John Berry.

\footnotetext{
${ }^{i}$ Merci à Elinor Ochs de m'avoir suggéré de donner des noms aux participants plutôt que d'utiliser des appellations SPK1 et SPK2.

${ }^{i i}$ Les conventions de transcription sont tirées du manuel CHAT.

iii Il aurait été possible par ailleurs d'analyser cette séquence en suivant l'étiquetage des récits (selon la typologie de Labov (1997), comme a pu le faire Ferré (2008) sur un récit humoristique dans le cadre d'un précédent CMLF), mais nous avons choisi de se concentrer sur l'emploi interactionnel et rhétorique des (dis)fluences.
} 